\title{
Spray-Dried Hierarchical Aggregates of Iron Oxide Nanoparticles and Their Functionalization for Downstream Processing in Biotechnology
}

\author{
Lennart Kleinfeldt, ${ }^{\dagger \ddagger}$ Johannes Gädke, ${ }^{\ddagger} \S$ Rebekka Biedendieck, ${ }^{\|, \perp}$ Rainer Krull, ${ }^{\ddagger}$, \\ and Georg Garnweitner*, $\uparrow, \ddagger$ (1) \\ ${ }^{\dagger}$ Institute for Particle Technology, Technische Universität Braunschweig, Volkmaroder Str. 5, 38104 Braunschweig, Germany \\ ${ }^{\ddagger}$ Center of Pharmaceutical Engineering-PVZ, Technische Universität Braunschweig, Franz-Liszt-Str. 35a, 38106 Braunschweig, \\ Germany \\ ${ }^{\S}$ Institute of Biochemical Engineering, Technische Universität Braunschweig, Rebenring 56, 38106 Braunschweig, Germany \\ "Institute of Microbiology, Technische Universität Braunschweig, Spielmannstr. 7, 38106 Braunschweig, Germany \\ ${ }^{\perp}$ Braunschweig Integrated Centre of Systems Biology (BRICS), Rebenring 56, 38106 Braunschweig, Germany
}

Supporting Information

\begin{abstract}
In this work, the structuring of iron oxide nanoparticles via spray-drying (SD) of aqueous suspensions is investigated, leading to micrometer-sized aggregates with saturation magnetization comparable to that of the individual nanoparticles. Interestingly, the superparamagnetic behavior is retained despite the multicore structure. Modification of the aggregates via the addition of silica nanoparticles to the suspension allows for control of the resulting magnetization by adjusting the iron oxide content. Moreover, the morphology of the produced aggregates is gradually shifted from irregular inflated-like shapes in case of pure iron oxide aggregates to reach spherical structures when bringing the silica content to only $20 \%$. The aggregates with different magnetization can be effectively separated in a simple column with an attached permanent magnet. Functionalization of pure iron oxide aggregates with a previously coupled ligand holding a nitrilotriacetic acid (NTA)-like moiety and subsequent loading with $\mathrm{Ni}^{2+}$ ions leads to the ability to bind 6-histidine ( $\left.\mathrm{His}_{6}\right)$-tagged target proteins via chelation complexes for magnetic separation. The application of the presented system for the purification of recombinant protein A in multiple cycles is shown. The recyclability of the separation system in combination with the high degree of magnetic separation is promising for future applications in the field of preparative in situ protein purification.
\end{abstract}

\section{INTRODUCTION}

Iron oxide nanoparticles are widely utilized in multiple fields such as the mining industry, biomedicine, and in applications for magnetic sealing using ferrofluids. ${ }^{1-5}$ They are established in various separation as well as purification tasks in food processing, wastewater treatment, and biotechnological production. ${ }^{3,6,7}$ In the size range below about $20 \mathrm{~nm}$, they contain a single domain and show superparamagnetism, ${ }^{8}$ which facilitates the handling in production processes since there is no magnetic attraction without a present external magnetic field. Based on the described characteristics, the term superparamagnetic iron oxide nanoparticles (SPIONs) is widely used. However, due to the small volume, the total magnetic moment of each individual particle is small and thus may require high-gradient magnetic separation systems for recovery. ${ }^{9,10}$ A bigger particle size, which could circumvent this shortcoming by an increased total magnetic moment, would in turn lead to a ferromagnetic behavior, making the general handling more difficult due to possible magnetic agglomer- ation. Therefore, magnetic nanoparticles are often encased by a polymer matrix or nonmagnetic shell of, for example, silica. ${ }^{11}$

The production of recombinant proteins and peptides plays an important role within the global biotechnology market with more than 400 marketed products. ${ }^{12}$ Thereby, downstream processing is responsible for up to $80 \%$ of the costs due to the standard use of multiple steps including chromatography. ${ }^{13,14}$ To reduce these costs, it is desirable to establish separation and purification strategies for continuous use. Various approaches using the adsorption onto magnetic nanoparticles and their subsequent separation have been reported..$^{15-17}$ The magnetic separation can be improved by increasing the magnetization of the individual particles, which is achieved by combining multiple nanoparticles in a multicore particle with a bigger size.

Received: May 27, 2019

Accepted: August 27, 2019

Published: September 26, 2019 
A

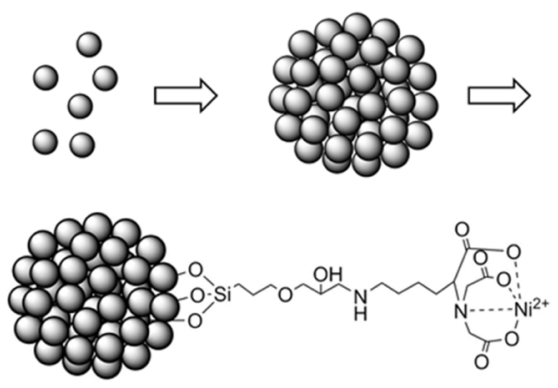

B

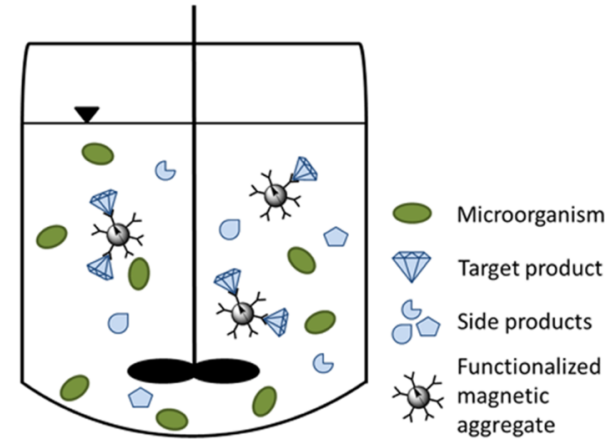

Figure 1. Schematic overview of (A) the spray-drying and functionalization process and (B) the application of the functionalized aggregates in protein purification.

Usually this is done by embedding SPIONs in a polymer matrix. $^{18}$

A different possibility to create multicore particles is the aggregation via spray-drying $(\mathrm{SD})$ of nanoparticulate suspensions. ${ }^{19} \mathrm{SD}$ is generally a well-established process with good reproducibility and scalability ${ }^{20}$ used in various industries where easier handling of particles and increased safety is reached via the granulation of nanoparticles. ${ }^{21}$ The size of aggregates that can be realized is limited to the lower micrometer range because of the combination of the typically low mass content of the used suspensions and a small droplet size that results from different parameters including the nozzle diameter. By SD of colloidal suspensions, structures containing multiple (iron oxide) cores can be prepared $^{22}$ and the morphology of the produced aggregates can be controlled. ${ }^{21}$ However, while iron oxide nanoparticles have been spray-dried in mixed materials, ${ }^{23-25}$ to the best of our knowledge, we present for the first time a systematic study on the SD of SPIONs and the resulting magnetic characteristics. Furthermore, spray-drying of a bimodal nanoparticle population in one suspension leads to the segregation of the particle classes during the drying process and results in core-shell-like aggregates with the smaller particles forming the shell and the bigger particle fraction being in the core based on a segregation process. ${ }^{26-28}$ The formation of a shell of small particles is only avoided when the drying time is drastically increased. ${ }^{28}$ Hence, by choosing an appropriate combination of process parameters as well as feed particles in terms of material characteristics and size, a tailored product can be prepared.

Our main goal in this study is to establish a tool for the targeted preparation of hierarchical magnetic aggregates with the option to tailor the magnetization and thus the magnetic separability of the resulting product. Moreover, the particles are modified for the continuous use in protein purification by providing a specific interaction and binding of a target protein in combination with repeated magnetic recovery and reusability. The material should offer a high specific surface area to maintain a large number of adsorption sites. Therefore iron oxide nanoparticles were synthesized via the nonaqueous sol-gel route and structured in a spray-drying process of aqueous suspensions to gain aggregates with increased magnetic recovery compared to nanoparticle agglomerates. The particles were functionalized with a tailored ligand for the specific interaction with the target product. The preparation of the functionalized SD-SPIONs as well as the concept of the application are schematically shown in Figure 1.

The synthesis and structuring are analyzed by various methods including X-ray diffraction (XRD), thermogravimetric analysis (TGA), laser diffraction, scanning electron microscopy (SEM), Fourier transform infrared spectroscopy (FT-IR), and magnetic analysis. Mixed aggregates with silica were analyzed in terms of their magnetization with a superconducting quantum interference device (SQUID), and the varied morphologies are demonstrated with electron microscopy.

While our previous study ${ }^{17}$ was focused on the integration of the in situ purification of proteins from microbial cultivation using agglomerated functionalized nanoparticles, here we focus on the tailoring of the magnetic particles to improve separability and retention over consecutive cycles.

\section{RESULTS AND DISCUSSION}

First, we characterized the structure and crystallinity of the prepared particles. The material can be identified as inverse spinel iron oxide from the powder X-ray diffractograms after synthesis as well as after spray-drying (Figure 2). However,

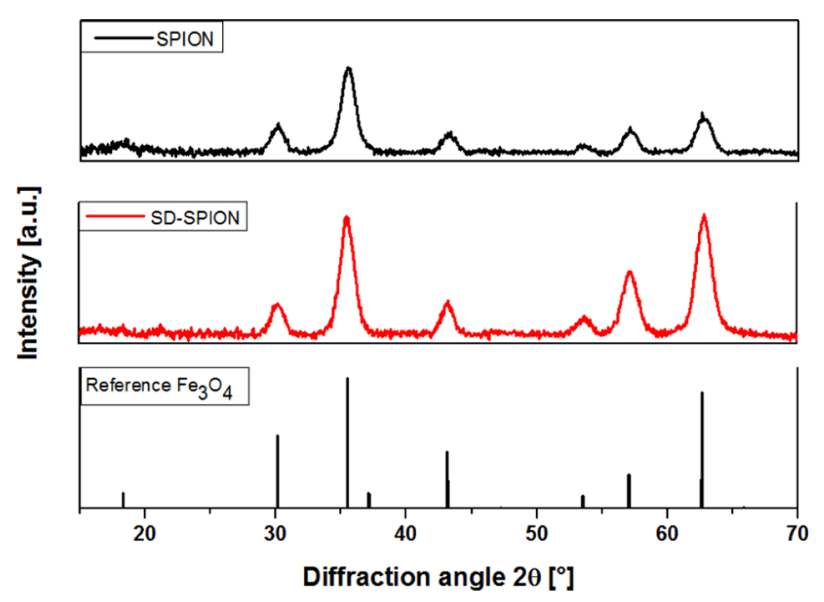

Figure 2. X-ray diffractograms of the synthesized iron oxide nanoparticles (SPIONs, top) and spray-dried aggregates (SDSPIONs, middle) with the reference pattern (bottom) of magnetite (ICSD-98-003-5000: $\mathrm{Fe}_{3} \mathrm{O}_{4}$ ).

maghemite and magnetite can hardly be distinguished due to the similarity of their respective crystal structures and consequently the corresponding diffraction patterns. ${ }^{29,30}$ Detailed investigations have revealed that the iron oxide particles under the used conditions typically form as mixed magnetitemaghemite nanocrystals both in hydrophobic and hydrophilic organic reaction media. ${ }^{31,32}$

Applying the Scherrer equation, the crystallite size of the examined samples can be calculated from the (311) reflection 
$\left(2 \theta=35.4^{\circ}\right)$ as the most pronounced signal. It is $6.5 \mathrm{~nm}$ for assynthesized particles and $6.6 \mathrm{~nm}$ for spray-dried particles, respectively. Interestingly, the spray-drying process does not lead to an increase of the size of the crystalline phases as indicated by the broad reflections. ${ }^{30}$ Hence, despite the formation of bonds between the individual nanoparticles during the aggregation process, a significant growth or ripening of the crystallites does not occur under the used conditions, most likely due to organic residues on the surface of the nanoparticles.

The particle size distribution of the obtained aggregates is given in Figure 3. The median diameter is $x_{50,3}=7.0 \mu \mathrm{m}$. A

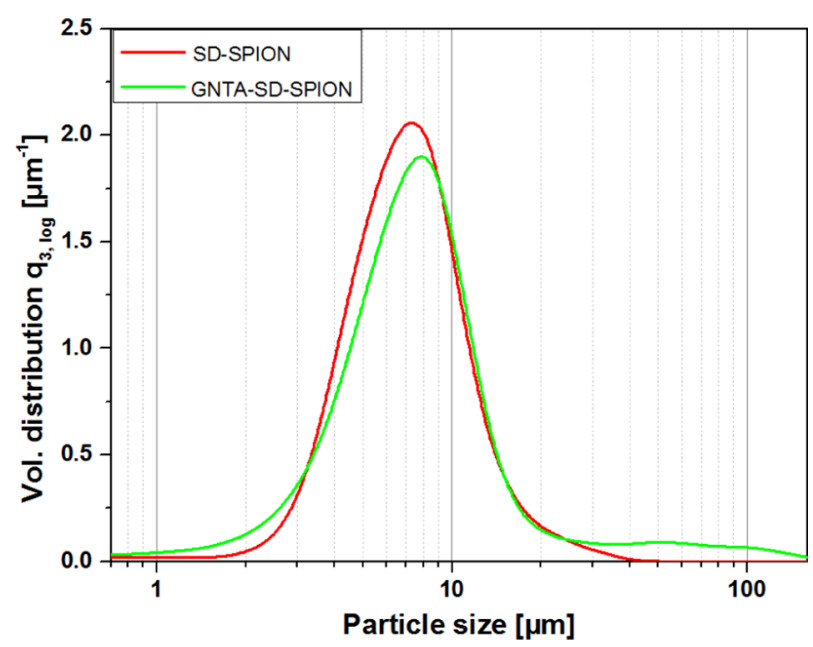

Figure 3. Aggregate particle size distribution of spray-dried pure iron oxide aggregates without (red) and with (green) GNTA-functionalization measured with laser diffraction in an aqueous suspension.

slight increase of the particle size to $7.4 \mu \mathrm{m}$ is observed after functionalization with fused ligand of GLYMO and L-NTA
(GNTA), which can be attributed to the occurrence of larger agglomerates that are possibly induced by interactions of the molecules of the added organic layer on the surface. Treatment of the aggregates with ultrasound does not lead to a change in the particle size. Consequently, the mechanical stability is expected to be high enough to withstand mechanical stresses that occur during biological applications such as microbial cultivation or separation processes.

The morphologies of pure iron oxide and mixed SD aggregates are depicted in Figure 4. Pure iron oxide aggregates show an irregular shape (Figure 4A) similar to doughnut-like or deflated structures caused by the buckling of a shell formed during the drying process that is influenced by cohesive and compressive forces in the drying droplet. ${ }^{21,33}$ The addition of $\mathrm{SiO}_{2}$ to the nanoparticle suspension results in a gradual change in the morphology (Figure 4B) and leads to spherical aggregates with $20 \% \mathrm{SiO}_{2}$ (Figure 4C) with an iron oxide shell around a silica core based on the segregation of the bimodal particles. ${ }^{28}$ The shape does not change when the $\mathrm{SiO}_{2}$ content is increased further (Figure 4D). The reason for this behavior is thought to be the different diffusion of particles during the drying of the droplets in the SD process. The small iron oxide particles will form a shell of the droplet with a thin, closely packed wall that remains stable during most of the drying process and leads to the formation of a hollow sphere that ultimately collapses, resulting in the buckling of the aggregates similar to the observation described by Sen et al. ${ }^{34}$ The introduction of bigger silica particles into the suspension changes the processes during the drying of the droplet, with the iron oxide wall being thinner and the particles able to reorganize while the droplet shrinks, thus leading to spherical aggregates. $^{23,33}$ Additionally, the surface of the aggregates shows increasing roughness with a higher content of the silica particles.

The mean diameters of aggregates with different iron oxide mass contents are given in Figure S2. The particle size is

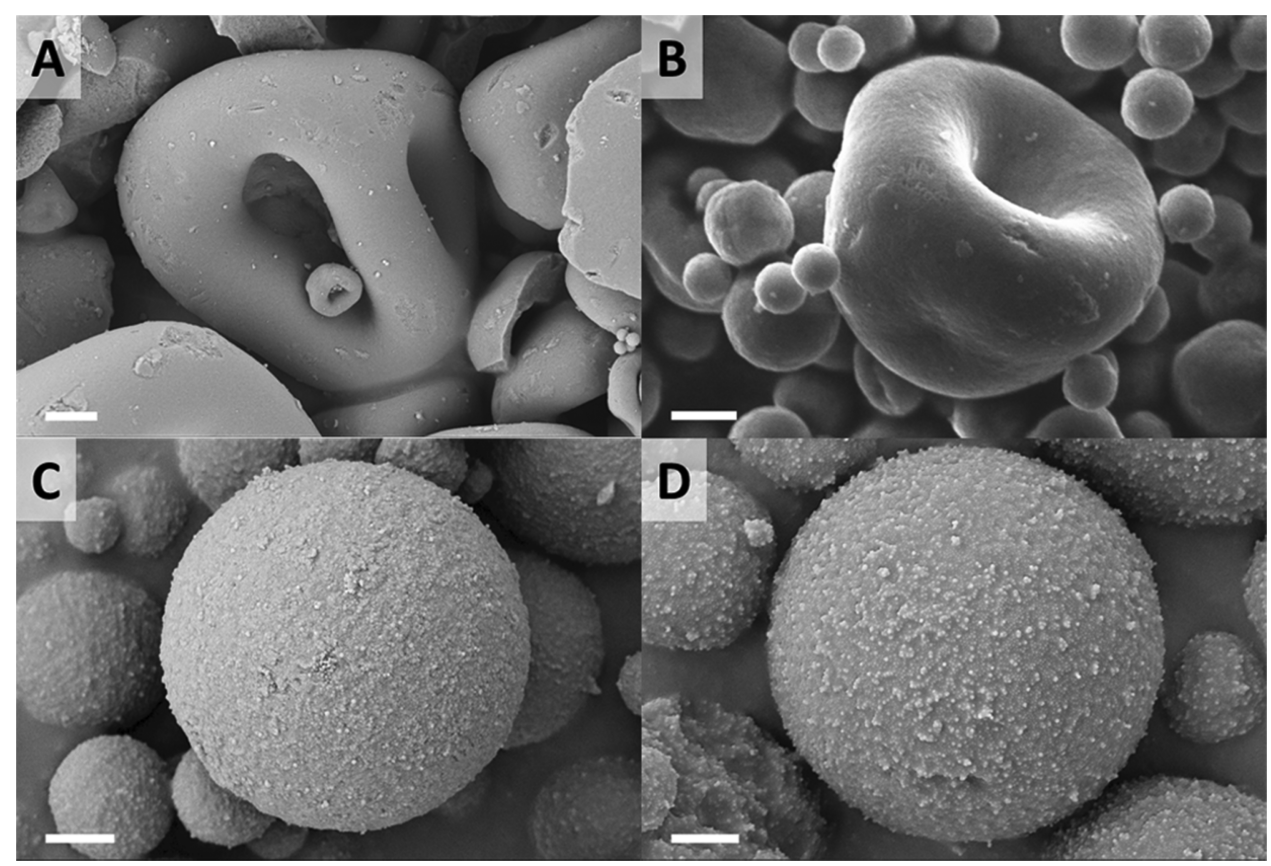

Figure 4. Scanning electron micrographs of aggregates: (A) SD-SPIONs; SD-SiO 2 -SPION-XX with iron oxide contents of (B) $90 \%$, (C) $80 \%$, and (D) $40 \%$, respectively. Scale bar is $2 \mu \mathrm{m}$ in all images. 
between 6 and $8 \mu \mathrm{m}$ for compositions within the range of 20$80 \% \mathrm{Fe}_{x} \mathrm{O}_{y}$. With further increasing mass content, the size increases to around $15 \mu \mathrm{m}$ for pure iron oxide aggregates.

Considering the previous observation, for aggregates with up to $80 \%$ iron oxide, there is a correlation between the spherical morphology and the consistent size of about $7 \mu \mathrm{m}$, while with increasing deviation from the spherical shape with a higher iron oxide content, the measured diameter shows a rising trend. The void volume in the deflated aggregates is not detected by light scattering and hence the overall size of the aggregates is derived from the outer diameter, which is bigger than that of a densely packed sphere.

Pure SD $\mathrm{Fe}_{x} \mathrm{O}_{y}$ has a specific surface area of $114.36 \mathrm{~m}^{2} \mathrm{~g}^{-1}$ as determined by nitrogen adsorption. The surface area of the asprepared nanoparticles cannot be directly measured in a dry state. However, assuming a particle size of $6.5 \mathrm{~nm}$ as obtained from XRD and a density of $5.2 \mathrm{~g} \mathrm{~m}^{-3}$, the specific surface area can be calculated to be $177.51 \mathrm{~m}^{2} \mathrm{~g}^{-1}$. Thus, the decrease in the specific surface area during the spray-drying process is relatively low, and a high porosity is present within the aggregates.

When measuring magnetization versus the magnetic flux density, the synthesized particles show no hysteresis loops, having neither remanence nor a coercive field, which is typical for superparamagnetic materials and is known for iron oxide particles below a diameter of $20 \mathrm{~nm}$ (Figure 5). Interestingly,

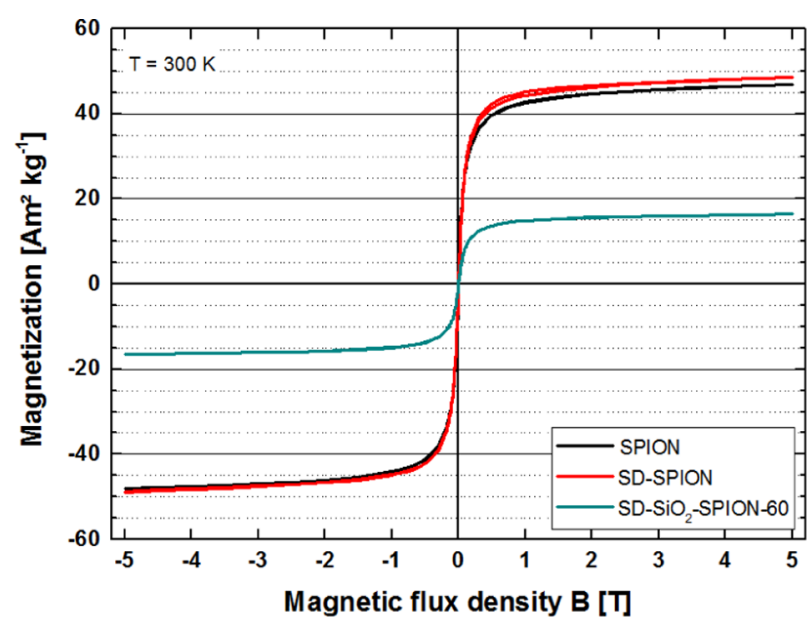

Figure 5. Magnetization curves of the synthesized iron oxide nanoparticles (black) and pure (red) as well as mixed (cyan) SD aggregates.

the spray-dried aggregates with a particle size of $7.0 \mu \mathrm{m}$ exhibit analogous superparamagnetic characteristics while showing a slightly increased saturation magnetization at $5 \mathrm{~T}$. Hence, the nanoparticles magnetically still behave like individual particles while there might be a positive interaction of the magnetic moments. The specific magnetization of the aggregates can be changed when the iron oxide nanoparticles are partially substituted by silica nanoparticles. The magnetization behavior without a hysteresis can still be observed, indicating that the iron oxide nanoparticles are not influenced in their magnetic characteristics.

The magnetization at $5 \mathrm{~T}$ of primary iron oxide nanoparticles as well as SD aggregates with various compositions is shown in Figure 6, with schematic illustrations of the morphology and the predicted internal aggregate structure. It

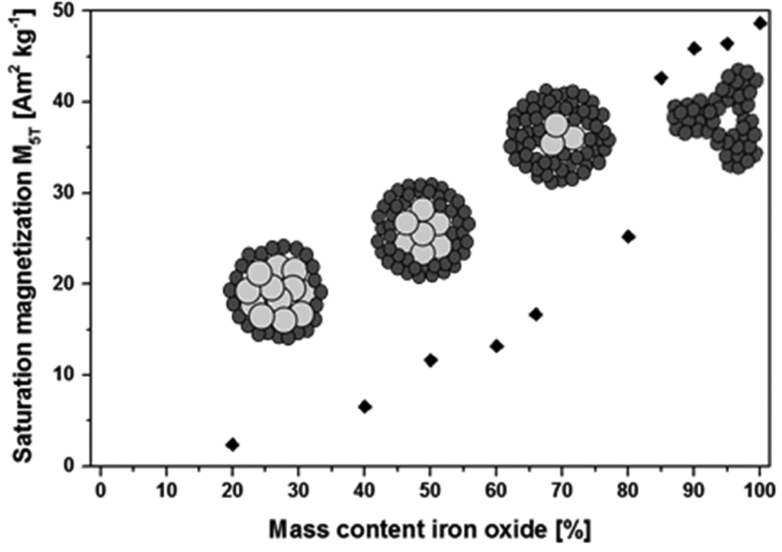

Figure 6. Magnetization at $5 \mathrm{~T}$ of spray-dried aggregates with a varying relative mass content of iron oxide. Drawings show the schematic aggregate structure consisting of SPIONs (dark gray) and silica nanoparticles (light gray).

can be clearly seen that there is not a linear but a sigmoidal dependency between the relative iron oxide mass content and the resulting magnetization $\mathrm{M}_{5 \mathrm{~T}}$ of the aggregates. A possible explanation is an increased interaction between the magnetic dipoles in the iron oxide nanoparticles within the thicker iron oxide shells that are formed with the increasing iron oxide mass content because of the particle segregation during the SD process. The observed enhancing effect of the magnetic properties of closely packed iron oxide nanoparticles has been reported in the literature based on increased magnetic interactions. $^{35,36}$

A possible application of the spray-dried aggregates is in protein purification using in situ affinity chromatography and magnetic separation. ${ }^{37}$ After successful separation, the adsorbed protein can be eluted from the particles and polished as a biotechnological product. To realize the selective binding of the particles with the target protein, the surface of the aggregates needs to be decorated with moieties allowing for a specific interaction. Here, the approach is to use NTA groups, which form complexation bonds with $\mathrm{His}_{6}$-tagged proteins and can be fused onto the particle surface with an appropriate functionalizing ligand that has been described previously. ${ }^{38,39}$

Iron oxide nanoparticles were analogously coupled to GNTA both directly after synthesis as well as after spraydrying to compare the success of functionalization. The amount of attached ligand was then analyzed by means of the mass added to the solid matter. Figure 7 presents a comparison of the relative mass during the heating of as-synthesized iron oxide particles and functionalized particles with and without structuring. All materials show a mass loss up to about $150{ }^{\circ} \mathrm{C}$, which can be attributed to the absorbed water. With further heating, organic compounds are desorbed from the particle surface. These can be both solvents from the synthesis that could not be washed off as evidenced for the primary particles (up to $300{ }^{\circ} \mathrm{C}$ ) and adsorbed ligands (up to $450{ }^{\circ} \mathrm{C}$ ) as seen in the functionalized particles, proving the success of this step. The distinct reduction in the total relative mass loss of the functionalized SD aggregates of about $10 \%$ in comparison to both the as-synthesized $(\approx 15 \%)$ and the functionalized nanoparticles $(\approx 35 \%)$ can be explained by the decreased specific surface of the aggregates, which results in less adsorbed organics per total mass. However, the theoretical decrease of specific surface is greater than the measured values. This could 


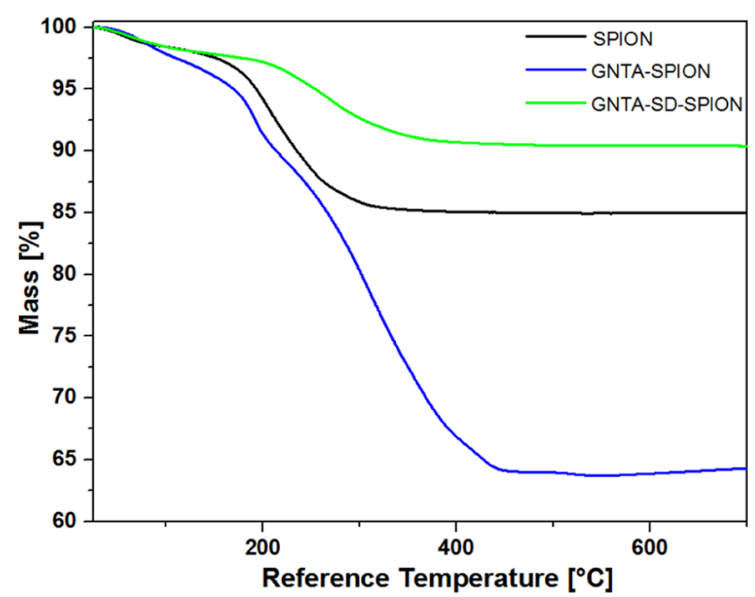

Figure 7. Thermogravimetric analysis of as-synthesized (SPIONs, black) and functionalized (GNTA-SPIONs, blue) iron oxide particles as well as functionalized spray-dried iron oxide aggregates (GNTASD-SPIONs, green).

be explained by the fact that the surface on the inside of the aggregates is inaccessible for the ligand molecules due to steric hindrance within the aggregate pore structure.

Nonetheless, FT-IR spectroscopy gives strong indications toward the successful coupling of the two individual molecules to form the bifunctional structure as well as the subsequent decoration of the particles with the as-prepared ligand also for the spray-dried particles (Figure 8 ). Besides the very strong

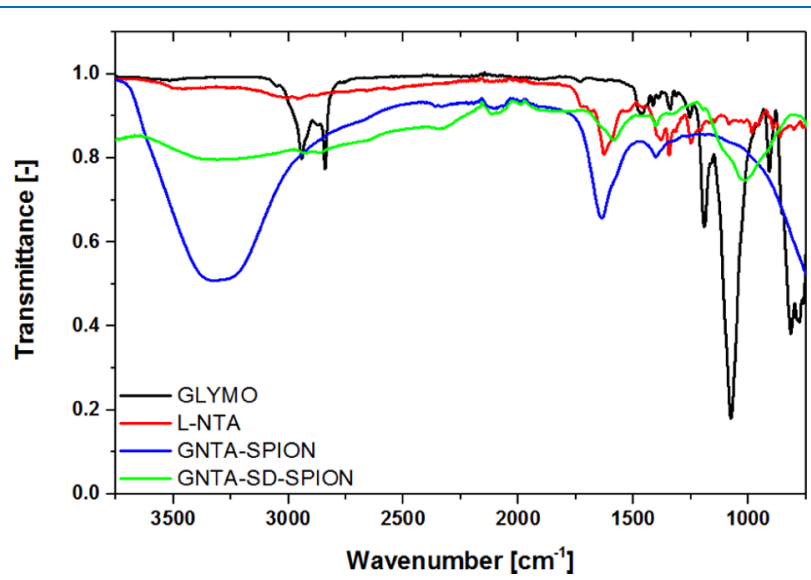

Figure 8. FT-IR transmittance of individual ligand molecules (GLYMO, black; L-NTA, red) and functionalized particles (GNTASPIONs, blue; GNTA-SD-SPIONs, green).

peak at $1075 \mathrm{~cm}^{-1}$ for the asymmetrical $\mathrm{Si}-\mathrm{O}-\mathrm{C}$ stretching vibration, the linker (3-glycidyloxypropyl)trimethoxysilane (GLYMO) shows two peaks typical for epoxy rings at 3052 $\mathrm{cm}^{-1}$ for the $\mathrm{C}-\mathrm{H}$ tension of the methylene group and at 908 $\mathrm{cm}^{-1}$ for the $\mathrm{C}-\mathrm{O}$ deformation vibration of the oxirane group prior to reaction. ${ }^{40}$ The broad peak in the range of 3500-3400 $\mathrm{cm}^{-1}$ indicates the stretching vibration, while the peak at 1630 $\mathrm{cm}^{-1}$ shows the deformation vibration of the $\mathrm{N}-\mathrm{H}$ bond of a primary amine $N_{\alpha}, N_{\alpha}$-bis (carboxymethyl)-L-lysine hydrate (LNTA) ${ }^{40}$ Upon coupling and consequently after functionalization, the peaks of the epoxy group disappear and those of the ethoxysilane group are greatly reduced, proving the crosslinking of the starting substances. ${ }^{41}$ Furthermore, the functionalized particles show a slight shift of the peak at $1630 \mathrm{~cm}^{-1}$ toward lower wavenumbers and an increase in the intensity of the peak at $1580 \mathrm{~cm}^{-1}$, indicating the presence of a secondary amine. $^{40}$

The magnetic separability of selected aggregates with different iron oxide-to-silica compositions was evaluated using the basic reactor setup presented by Gädke et al. ${ }^{42}$ For the basic separation efficiency experiments, an abiotic system was used, suspending the nonfunctionalized particles in deionized water. The complete reactor volume was cycled through the external loop seven times to ensure comparability with the previous findings. The results are presented in Table 1 , showing that even when halving the content of magnetic

Table 1. Magnetic Separation of Spray-Dried Particles with Different Iron Oxide Contents

$\begin{array}{cc}\text { sample mass content iron oxide (\%) } & \text { magnetic separation efficiency (\%) } \\ 50.0 & 90.3 \\ 66.6 & 92.1 \\ 85.0 & 92.4 \\ 100.0 & 93.0^{a}\end{array}$

${ }^{a}$ Measured in different cultivation systems (shake flask) using identical magnets.

$\mathrm{Fe}_{x} \mathrm{O}_{y}$ nanoparticles in the aggregates, the threshold of $90 \%$ separation efficiency is reached. Hence, the use of these aggregates in further applications is promising to be a lot more cost-efficient in direct comparison with the pure SD-SPIONs considering the inexpensive synthesis of silica nanoparticles compared to the nonaqueous preparation of the $\mathrm{Fe}_{x} \mathrm{O}_{y}$ nanoparticles utilized here.

Additionally, it was shown that the recovery of magnetic particles can be increased in comparison to functionalized SPIONs due to the improved magnetic separability of the aggregates. When GNTA-SPIONs are applied in the in situ purification in shaking flasks, an average particle loss of approximately $30 \%$ per cycle over five consecutive cycles is detected. ${ }^{17}$ In contrast, on average, $93 \%$ of the GNTA-SDSPIONs are retained during the separation process in shaking flasks.

The purity of the product was determined after each separation step as it is crucial for its evaluation. Figure 9 shows the sodium dodecyl sulfate polyacrylamide gel electrophoresis (SDS-PAGE) of recombinant protein A after elution from regenerated GNTA-SD-SPIONs. Duplicates after cycles 3, 4, and 5 as well as a protein size reference are depicted. The separated product shows a protein lane at approximately 27 $\mathrm{kDa}$ as expected. The second line that can be seen at around

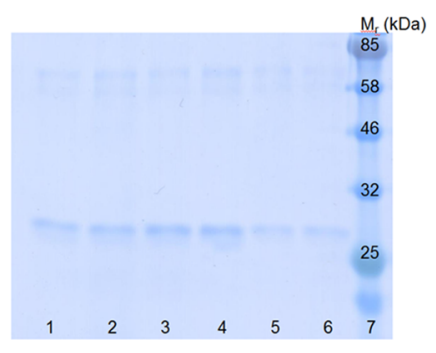

Figure 9. SDS-PAGE of elution fractions containing protein A after separation with GNTA-SD-SPIONs in multiple cycles (lanes 1 and 2, cycle 3; lanes 3 and 4, cycle 4; lanes 5 and 6 , cycle 5; lane 7, protein size reference). 
$60 \mathrm{kDa}$ can be attributed to agglomerates caused by the high concentration during the precipitation with ammonium sulfate used to enhance sensitivity. Assuming the bands at $\approx 60 \mathrm{kDa}$ to be agglomerates of the protein, the purity of protein $\mathrm{A}$ is calculated to be $88.2 \pm 9.4 \%$ with regard to protein compounds.

The integrated automation of a lab-scale stirred-tank reactor for the production, in situ separation, and purification of recombinant proteins utilizing the functionalized spray-dried pure SPION aggregates was reported by Gädke et al. ${ }^{42}$

\section{CONCLUSIONS}

We presented the successful synthesis of SPIONs and their structuring in a spray-drying process. Moreover, the straightforward possibility to tailor the morphology and magnetic properties of the aggregates by adjusting the ratio of SPIONs and silica nanoparticles in the feed suspension was demonstrated. On the one hand, the morphology of the resulting aggregates changed from irregularly shaped, inflated spheres with a very high iron oxide content to spherical structures when the silica content was increased to $20 \%$ or higher. On the other hand, the magnetic behavior of the product remained superparamagnetic, similar to the primary iron oxide nanoparticles despite the size of the aggregates being several hundred single-core diameters. Additionally, the saturation magnetization is slightly enhanced compared to that of primary SPIONs. Hence, we conclude that the SPIONs were still sufficiently separated within the structured particle to avoid coalescence of the magnetic cores, while enabling positive magnetic interaction. In a subsequent step, the functionalization of pure iron oxide aggregates analogous to a previously reported nanoparticle modification was successfully performed to achieve a highly selective but reversible coupling of tagged proteins, although the reduction of the specific surface area and thus the number of ligand molecules that can be attached to the particles leads to a smaller amount of sites for protein binding. The magnetic separability of aggregates with differing silica contents was proven in an abiotic setup, revealing a separation efficiency $>90 \%$ even with a magnetic particle content of only 50\%. The increased magnetic separability in comparison to individual nanoparticles is expected to overcompensate the reduction of active binding sites in the presented application of protein separation. Finally, the functionalized SD iron oxide aggregates were successfully used in affinity separation processes to purify recombinant proteins. Here, the spray-dried GNTA-SD-SPIONs showed superiority over nonstructured GNTA-SPIONs due to their defined structure, which remains unchanged throughout several recovery cycles, and their fast and facile magnetic separation. Therefore, the presented system shows substantial advantages with regard to multicycle reusability and is highly promising for preparative applications in biotechnology also at larger scales.

\section{EXPERIMENTAL SECTION}

Materials. Iron(III) acetylacetonate (99.9\%), triethylene glycol (ReagentPlus, 99.0\%), (3-glycidyloxypropyl)trimethoxysilane (98.0\%) (GLYMO), $N_{\alpha}, N_{\alpha}$-bis (carboxymethyl)-L-lysine hydrate (97.0\%) (L-NTA, so-called due to the structural similarity with nitrilotriacetic acid (NTA)), tetraethyl orthosilicate (TEOS), $\mathrm{NaOH}$, and $\mathrm{HCl}$ were purchased from Sigma-Aldrich and were used as received.
Deionized water (in-house reverse-osmosis equipment) was used for all experiments.

Synthesis. Superparamagnetic iron oxide nanoparticles (SPIONs) were synthesized in a benchtop reactor (Model 4568, Parr Instrument Company, Moline, IL) according to Grabs et al. ${ }^{43}$ using iron(III) acetylacetonate as a metalorganic precursor and triethylene glycol as the solvent. The reaction was conducted for $24 \mathrm{~h}$ at $200{ }^{\circ} \mathrm{C}$. The product was washed three times with ethyl acetate and transferred to deionized water for further processing or dried in a desiccator for analysis purposes. The primary particles that are obtained after synthesis are of spherical morphology with a particle size of 6-7 nm in agreement with Masthoff et al. (data not shown). ${ }^{32}$

$\mathrm{SiO}_{2}$ particles were synthesized applying the Stöber process $^{26,44}$ in a glass reactor (Büchi AG, Uster, Switzerland) at a reaction temperature of $6{ }^{\circ} \mathrm{C}$ by adding $101 \mathrm{~mL}$ of TEOS to a solution of ammonium hydroxide $(10.1 \mathrm{~mL})$ in $602 \mathrm{~mL}$ of ethanol and $76.8 \mathrm{~mL}$ of deionized water and allowing to react for $24 \mathrm{~h}$. The silica particles have a median diameter $x_{50,3}$ of approximately $200 \mathrm{~nm}$ (Supporting Information Figure S1).

Spray-Drying of Iron Oxide Nanoparticles. Aggregates were prepared by spray-drying an aqueous nanoparticle suspension with $5 \% \mathrm{w} / \mathrm{w}$ solid content of iron oxide nanoparticles (Figure 1A, top). The spray-drying process conditions in the used lab-scale spray-dryer (4M8-Trix, ProCepT Inc., Zelzate, Belgium) were a drying temperature of $100{ }^{\circ} \mathrm{C}$, a pump rate of $2.96 \mathrm{~mL} \mathrm{~min}^{-1}$, and an air speed of $0.30 \mathrm{~m}^{3} \mathrm{~min}^{-1}$ using a two-fluid nozzle with a diameter of 0.8 $\mu \mathrm{m}$. The obtained product is denoted as SD-SPION.

Spray-Drying of Mixtures of Iron Oxide and Silica Nanoparticles. To investigate the possibility to tailor the magnetization of the SD aggregates, the composition of the suspensions was changed using a mixture of varying amounts of iron oxide and silica particles, while maintaining the total solid content at a constant level. Silica particles (size $200 \mathrm{~nm}$ ) were spray-dried with the previously described SPIONs. The spray-drying conditions were kept constant in comparison to the pure iron oxide spray-drying process. The obtained products were denoted as $\mathrm{SD}-\mathrm{SiO}_{2}-\mathrm{SPION}-\mathrm{XX}$, giving the iron oxide nanoparticle content expressed as a percentage, for example, $\mathrm{SD}-\mathrm{SiO}_{2}$-SPION-90 with $90 \%$ iron oxide and $10 \%$ silica nanoparticles, respectively.

Ligand Coupling. The desired binding functionality with the target proteins was accomplished by using the capability of groups structurally similar to NTA to build chelation bonds with divalent metal ions such as $\mathrm{Ni}^{2+}$. The ligand was prepared as described by Masthoff et al. by coupling GLYMO and LNTA, being structurally similar to NTA, to obtain GNTA. ${ }^{39}$ Briefly, $1.41 \mathrm{~g}$ of L-NTA was added to a solution of $14.13 \mathrm{~mL}$ of $\mathrm{NaOH}(10 \mathrm{M})$ and $25.00 \mathrm{~mL}$ of deionized water. This solution was cooled to $0{ }^{\circ} \mathrm{C}$ in an ice bath before $1.19 \mathrm{~mL}$ of GNTA was added dropwise under rigorous stirring. After subsequent warm-up to room temperature within $5 \mathrm{~h}$, the reaction mixture was heated to $65{ }^{\circ} \mathrm{C}$ and stirred overnight. Subsequently, the $\mathrm{pH}$ of the resulting solution was adjusted to 11 by adding hydrogen chloride $(6 \mathrm{M})$ prior to further processing.

Functionalization. The prepared pure iron oxide SDSPIONs were functionalized with the GNTA-ligand (Figure $1 \mathrm{~A}$, bottom) adapting the method described by Masthoff et al. ${ }^{39}$ Since the SD aggregates are prone to sedimentation, they have to be agitated during the reaction to ensure complete 
functionalization, which was realized in a benchtop reactor (Model 4568, Parr Instrument Company, Moline, IL) under stirring at $150 \mathrm{~min}^{-1}$. The ligand solution $(8.6 \mathrm{~mL})$ was used after adjusting $\mathrm{pH}=11$. Then, SD-SPIONs were suspended in deionized water to a concentration of $10 \mathrm{mg} \mathrm{mL}^{-1}$ and added to the ligand solution. Subsequently, the suspension was heated to $95{ }^{\circ} \mathrm{C}$ for $4 \mathrm{~h}$ and afterward left to cool down to room temperature overnight. Afterward, the aggregates were washed thoroughly with deionized water and dried in a desiccator for storage.

Application. The cultivation, synthesis, and purification of the model protein with functionalized SPIONs have been presented in a previous study ${ }^{17}$ and thus will only be described shortly. Protein A was recombinantly produced and secreted from Bacillus megaterium MS 941 that was transformed with a recombinant plasmid encoding a truncated protein $A$ with a histidine 6-tag $\left(\mathrm{His}_{6}\right)$ at the C-terminal end. ${ }^{17,45}$ The bacterial strain was cultivated in a defined medium at $37^{\circ} \mathrm{C}$ in a shaking flask as a preculture and inoculated for production in a DASGIP bioreactor (TC4 SC4; MX4/4; PH4 PO4; CWD 4; MP8, DASGIP Information and Process Technology, Jülich, Germany), adding xylose $\left(5 \mathrm{~g} \mathrm{~L}^{-1}\right)$ to induce the production (cultivation parameters in Table S1). The in situ separation of the recombinant protein (concentration reached, $90 \mathrm{mg} \mathrm{mL}^{-1}$ ) from the growth medium occurred during the exponential growth phase when functionalized particles were added to a final concentration of $0.6 \mathrm{mg} \mathrm{mL}^{-1}$ and magnetically separated after $10 \mathrm{~min}$ by pumping the medium through an external loop with an attached permanent magnet. ${ }^{42}$ The application concept is depicted in Figure 1B. Subsequently, the loaded particles were washed, and recombinant proteins were eluted from the particles with appropriate buffers (compare Tables S2 and S3) before the particles were regenerated and collected to be reloaded with $\mathrm{Ni}^{2+}$ (see Tables S4 and S5) to regain the specific absorption capacity. Eluted proteins were analyzed by sodium dodecyl sulfate polyacrylamide gel electrophoresis (SDS-PAGE) as described previously. ${ }^{17}$ Finally, after further cultivation, the particles were added back into the bioreactor for an additional separation cycle.

Characterization. X-ray diffraction (XRD) measurements were conducted using an EMPYREAN device (PANalytical B.V., Almelo, The Netherlands) at a wavelength of $K_{\alpha}=0.154$ $\mathrm{nm}$ with copper radiation (Empyrean Cu LFF HR) in a range of $2 \theta$ from 15 to $70^{\circ}$ and a step size of $0.05^{\circ}$ (PIXcel-3D detector, Malvern Panalytical Ltd., Malvern, U.K.).

Particle size analysis of the SD aggregates both with and without functionalization was conducted on a HELOS/BF laser diffraction device (Sympatec $\mathrm{GmbH}$, Clausthal-Zellerfeld, Germany) equipped with a wet dispersing system CUVETTE. The sample was dispersed in water and stirred manually. For the initial evaluation of the mechanical integrity, the sample was stressed with ultrasonication (US) using the built-in USfinger at $100 \%$ intensity for $30 \mathrm{~s}$.

The morphology of spray-dried aggregates was analyzed using scanning electron microscopy (SEM). SEM images were taken with an LEO 1550 device (Carl Zeiss Microscopy $\mathrm{GmbH}$, Jena, Germany).

The magnetic properties of the as-synthesized iron oxide nanoparticles as well as all SD aggregates were analyzed with a superconducting quantum interference device (SQUID). The SQUID measurements were conducted with a Magnetic Property Measurement System (Quantum Design Inc., San
Diego, CA). The magnetization was measured at $300 \mathrm{~K}$, and the magnetic flux density was varied between -5 and $5 \mathrm{~T}$.

The specific surface areas of the prepared aggregates of pure iron oxide were analyzed with a Nova 2000e Surface Area \& Pore Size Analyzer (Quantachrome $\mathrm{GmbH} \&$ Co. KG, Odelzhausen, Germany).

The amount of functionalization agent attached to the particle surface was investigated by applying thermogravimetric analysis. The sample under investigation $(15 \mathrm{mg})$ was heated from 25 to $700{ }^{\circ} \mathrm{C}$ with a constant heating rate of $10{ }^{\circ} \mathrm{C} \mathrm{min}^{-1}$. A TGA/DSC 1 STAR $^{\mathrm{e}}$ system (Mettler-Toledo AG, Schwerzenbach, Switzerland) was used for the investigation. The coupling of the ligand as well as its binding onto the surface of the spray-dried aggregates was evaluated via Fourier transform infrared spectroscopy (FT-IR) with Vertex 70 (Bruker Corporation, Billerica, MA). The magnetic separability of selected SD aggregates was tested using aqueous suspensions in a laboratory setup of a circulation loop with handheld magnets as described elsewhere. ${ }^{42}$

\section{ASSOCIATED CONTENT}

\section{Supporting Information}

The Supporting Information is available free of charge on the ACS Publications website at DOI: 10.1021/acsomega.9b01549.

Particle size analysis of silica nanoparticles; mean aggregate sizes with varying silica contents; microbial cultivation parameters; compositions of all used buffers (PDF)

\section{AUTHOR INFORMATION}

\section{Corresponding Author}

*E-mail: g.garnweitner@tu-braunschweig.de.

ORCID

Georg Garnweitner: 0000-0002-7499-4947

\section{Author Contributions}

L.K. and G.G. conceived the study. L.K. performed all synthesis, spray-drying, and functionalization experiments and the corresponding analyses as well as prepared figures and the manuscript. R.B. provided the biological system for recombinant protein A production. J.G. conducted the cultivation work and protein analysis. R.K. and G.G. supervised the study and participated in its design and coordination. All authors helped draft and have given approval to the final version of the manuscript.

\section{Notes}

The authors declare no competing financial interest.

\section{ACKNOWLEDGMENTS}

The authors acknowledge Dr. D. Menzel (Institute of Condensed Matter Physics) for executing SQUID measurements and P. Pfeiffer (Institute for Materials) for taking SEM images, both from Technische Universität Braunschweig, Germany. This work was financially supported by the Lower Saxony Ministry for Science and Culture within the research group ElektroBak - Innovative materials and concepts for microbial electrochemical systems (L.K.) as well as in the joint research project Novel synthesis and formulation methods for poorly soluble drugs and sensitive biopharmaceuticals (SynFoBiA) (J.G.) within the Center of Pharmaceutical Engineering (PVZ) at the Technische Universität Braunschweig, Germany. We 
acknowledge support by the German Research Foundation and the Open Access Publication Funds of the Technische Universität Braunschweig.

\section{ABBREVIATIONS}

$\mathrm{His}_{6}$, 6-histidine

FT-IR, Fourier transform infrared spectroscopy

GLYMO, (3-glycidyloxypropyl)trimethoxysilane

GNTA, fused ligand of GLYMO and L-NTA

L-NTA, $N_{\alpha}, N_{\alpha}$-bis(carboxymethyl)-L-lysine hydrate

NTA, nitrilotriacetic acid

$\mathrm{SD}-\mathrm{SiO}_{2}$-SPION, spray-dried silica superparamagnetic iron oxide nanoparticle aggregate

SDS-PAGE, sodium dodecyl sulfate polyacrylamide gel electrophoresis

SD-SPION, spray-dried superparamagnetic iron oxide nanoparticle aggregate

SEM, scanning electron microscopy

SPION, superparamagnetic iron oxide nanoparticle

SQUID, superconductive quantum interference device

TEOS, tetraethyl orthosilicate

TGA, thermogravimetric analysis

$\mathrm{XRD}, \mathrm{X}$-ray diffractometry

\section{REFERENCES}

(1) Mandel, K.; Hutter, F. The magnetic nanoparticle separation problem. Nano Today 2012, 7, 485-487.

(2) Nguyen, T.-D. From formation mechanisms to synthetic methods toward shape-controlled oxide nanoparticles. Nanoscale 2013, 5, 9455-9482.

(3) Bao, Y.; Wen, T.; Samia, A. C. S.; Khandhar, A.; Krishnan, K. M. Magnetic nanoparticles: material engineering and emerging applications in lithography and biomedicine. J. Mater. Sci. 2016, 51, 513553.

(4) Shen, W.-Z.; Cetinel, S.; Sharma, K.; Borujeny, E. R.; Montemagno, C. Peptide-functionalized iron oxide magnetic nanoparticle for gold mining. J. Nanopart. Res. 2017, 19, 74.

(5) Teja, A. S.; Koh, P.-Y. Synthesis, properties, and applications of magnetic iron oxide nanoparticles. Prog. Cryst. Growth Charact. Mater. 2009, 55, 22-45.

(6) Faraji, M.; Yamini, Y.; Rezaee, M. Magnetic nanoparticles: Synthesis, stabilization, functionalization, characterization and applications. J. Iran. Chem. Soc. 2010, 7, 1-37.

(7) Frey, N. A.; Peng, S.; Cheng, K.; Sun, S. Magnetic nanoparticles: synthesis, functionalization, and applications in bioimaging and magnetic energy storage. Chem. Soc. Rev. 2009, 38, 2532-2542.

(8) Liu, X. L.; Fan, H. M. Innovative magnetic nanoparticle platform for magnetic resonance imaging and magnetic fluid hyperthermia applications. Curr. Opin. Chem. Eng. 2014, 4, 38-46.

(9) Fraga García, P.; Brammen, M.; Wolf, M.; Reinlein, S.; Freiherr von Roman, M.; Berensmeier, S. High-gradient magnetic separation for technical scale protein recovery using low cost magnetic nanoparticles. Sep. Purif. Technol. 2015, 150, 29-36.

(10) Lindner, J.; Nirschl, H. A hybrid method for combining HighGradient Magnetic Separation and centrifugation for a continuous process. Sep. Purif. Technol. 2014, 131, 27-34.

(11) Cao, G.; Gao, J.; Zhou, L.; Huang, Z.; He, Y.; Zhu, M.; Jiang, Y. Fabrication of $\mathrm{Ni}^{2+}$-nitrilotriacetic acid functionalized magnetic mesoporous silica nanoflowers for one pot purification and immobilization of His-tagged $\omega$-transaminase. Biochem. Eng. J. 2017, $128,116-125$.

(12) Sanchez-Garcia, L.; Martín, L.; Mangues, R.; Ferrer-Miralles, N.; Vázquez, E.; Villaverde, A. Recombinant pharmaceuticals from microbial cells: a 2015 update. Microb. Cell Fact. 2016, 15, 33.

(13) Gagnon, P. Technology trends in antibody purification. J. Chromatogr. A 2012, 1221, 57-70.
(14) Roque, A. C. A.; Lowe, C. R.; Taipa, M. A. Antibodies and genetically engineered related molecules: production and purification. Biotechnol. Prog. 2004, 20, 639-654.

(15) Franzreb, M.; Siemann-Herzberg, M.; Hobley, T. J.; Thomas, O. R. T. Protein purification using magnetic adsorbent particles. Appl. Microbiol. Biotechnol. 2006, 70, 505-516.

(16) Fraga García, P.; Freiherr von Roman, M.; Reinlein, S.; Wolf, M.; Berensmeier, S. Impact of nanoparticle aggregation on protein recovery through a pentadentate chelate ligand on magnetic carriers. ACS Appl. Mater. Interfaces 2014, 6, 13607-13616.

(17) Gädke, J.; Kleinfeldt, L.; Schubert, C.; Rohde, M.; Biedendieck, R.; Garnweitner, G.; Krull, R. In situ affinity purification of his-tagged protein A from Bacillus megaterium cultivation using recyclable superparamagnetic iron oxide nanoparticles. J. Biotechnol. 2017, 242, $55-63$.

(18) Sommertune, J.; Sugunan, A.; Ahniyaz, A.; Bejhed, R. S.; Sarwe, A.; Johansson, C.; Balceris, C.; Ludwig, F.; Posth, O.; Fornara, A. Polymer/Iron Oxide Nanoparticle Composites-A Straight Forward and Scalable Synthesis Approach. Int. J. Mol. Sci. 2015, 16, 1975219768.

(19) Walton, D. E.; Mumford, C. J. The Morphology of Spray-Dried Particles. Chem. Eng. Res. Des. 1999, 77, 442-460.

(20) Sosnik, A.; Seremeta, K. P. Advantages and challenges of the spray-drying technology for the production of pure drug particles and drug-loaded polymeric carriers. Adv. Colloid Interface Sci. 2015, 223, $40-54$.

(21) Lintingre, E.; Lequeux, F.; Talini, L.; Tsapis, N. Control of particle morphology in the spray drying of colloidal suspensions. Soft Matter 2016, 12, 7435-7444.

(22) Zhou, G.-W.; Wang, J.; Gao, P.; Yang, X.; He, Y.-S.; Liao, X.-Z.; Yang, J.; Ma, Z.-F. Facile spray drying route for the three-dimensional graphene-encapsulated $\mathrm{Fe}_{2} \mathrm{O}_{3}$ nanoparticles for lithium ion battery anodes. Ind. Eng. Chem. Res. 2013, 52, 1197-1204.

(23) Ehrhart, B. D.; Ward, B. J.; Richardson, B. M.; Anseth, K. S.; Weimer, A. W. Partial flocculation for spray drying of spherical mixed metal oxide particles. J. Am. Ceram. Soc. 2018, 101, 4452-4457.

(24) Huang, H.-Y.; Shieh, Y.-T.; Shih, C.-M.; Twu, Y.-K. Magnetic chitosan/iron (II, III) oxide nanoparticles prepared by spray-drying. Carbohydr. Polym. 2010, 81, 906-910.

(25) Pfeiffer, S.; Florio, K.; Makowska, M.; Ferreira Sanchez, D.; van Swygenhoven, H.; Aneziris, C. G.; Wegener, K.; Graule, T. Iron Oxide Doped Spray Dried Aluminum Oxide Granules for Selective Laser Sintering and Melting of Ceramic Parts. Adv. Eng. Mater. 2019, 21, No. 1801351.

(26) Zellmer, S.; Garnweitner, G.; Breinlinger, T.; Kraft, T.; Schilde, C. Hierarchical structure formation of nanoparticulate spray-dried composite aggregates. ACS Nano 2015, 9, 10749-10757.

(27) Jabłczyńska, K.; Gac, J. M.; Sosnowski, T. R. Self-organization of colloidal particles during drying of a droplet: Modeling and experimental study. Adv. Powder Technol. 2018, 29, 3542-3551.

(28) Liu, W.; Midya, J.; Kappl, M.; Butt, H.-J.; Nikoubashman, A. Segregation in Drying Binary Colloidal Droplets. ACS Nano 2019, 13, 4972-4979.

(29) Kim, W.; Suh, C.-Y.; Cho, S.-W.; Roh, K.-M.; Kwon, H.; Song, K.; Shon, I.-J. A new method for the identification and quantification of magnetite-maghemite mixture using conventional X-ray diffraction technique. Talanta 2012, 94, 348-352.

(30) Cornell, R. M.; Schwertmann, U. The Iron Oxides: Structure, Properties, Reactions, Occurrences, and Uses, 2nd, completely rev. and extended ed.; Wiley-VCH: Weinheim, 2003.

(31) Masthoff, I.-C.; Kraken, M.; Mauch, D.; Menzel, D.; Munevar, J. A.; Baggio Saitovitch, E.; Litterst, F. J.; Garnweitner, G. Study of the growth process of magnetic nanoparticles obtained via the nonaqueous sol-gel method. J. Mater. Sci. 2014, 49, 4705-4714.

(32) Masthoff, I.-C.; Kraken, M.; Menzel, D.; Litterst, F. J.; Garnweitner, G. Study of the growth of hydrophilic iron oxide nanoparticles obtained via the non-aqueous sol-gel method. J. Sol-Gel Sci. Technol. 2016, 77, 553-564. 
(33) Breinlinger, T.; Hashibon, A.; Kraft, T. Simulation of the Spray Drying of Single Granules: The Correlation Between Microscopic Forces and Granule Morphology. J. Am. Ceram. Soc. 2015, 98, 17781786.

(34) Sen, D.; Bahadur, J.; Mazumder, S.; Verma, G.; Hassan, P. A.; Bhattacharya, S.; Vijai, K.; Doshi, P. Nanocomposite silicasurfactant microcapsules by evaporation induced self assembly: tuning the morphological buckling by modifying viscosity and surface charge. Soft Matter 2012, 8, 1955-1963.

(35) Dennis, C. L.; Jackson, A. J.; Borchers, J. A.; Ivkov, R.; Foreman, A. R.; Lau, J. W.; Goernitz, E.; Gruettner, C. The influence of collective behavior on the magnetic and heating properties of iron oxide nanoparticles. J. Appl. Phys. 2008, 103, No. 07A319.

(36) Smolkova, I. S.; Kazantseva, N. E.; Babayan, V.; Vilcakova, J.; Pizurova, N.; Saha, P. The Role of Diffusion-Controlled Growth in the Formation of Uniform Iron Oxide Nanoparticles with a Link to Magnetic Hyperthermia. Cryst. Growth Des. 2017, 17, 2323-2332.

(37) Berensmeier, S. Magnetic particles for the separation and purification of nucleic acids. Appl. Microbiol. Biotechnol. 2006, 73, 495-504.

(38) Birger Anspach, F. Silica-based metal chelate affinity sorbents I. Preparation and characterization of iminodiacetic acid affinity sorbents prepared via different immobilization techniques. $J$. Chromatogr. A 1994, 672, 35-49.

(39) Masthoff, I.-C.; David, F.; Wittmann, C.; Garnweitner, G. Functionalization of magnetic nanoparticles with high-binding capacity for affinity separation of therapeutic proteins. J. Nanopart. Res. 2014, 16, 1-10.

(40) González, M. G.; Cabanelas, J. C.; Baselga, J. Applications of FTIR on Epoxy Resins - Identification, Monitoring the Curing Process, Phase Separation and Water Uptake. In Infrared Spectroscopy - Materials Science, Engineering and Technology; Theophile, T., Ed.; InTech, 2012.

(41) Nikolic, G.; Zlatkovic, S.; Cakic, M.; Cakic, S.; Lacnjevac, C.; Rajic, Z. Fast Fourier transform IR characterization of epoxy GY systems crosslinked with aliphatic and cycloaliphatic EH polyamine adducts. Sensors 2010, 10, 684-696.

(42) Gädke, J.; Thies, J.-W.; Kleinfeldt, L.; Kalinin, A.; Starke, G.; Lakowitz, A.; Biedendieck, R.; Garnweitner, G.; Dietzel, A.; Krull, R. Integrated in situ -purification of recombinant proteins from Bacillus megaterium cultivation using SPION in stirred tank reactors. Biochem. Eng. J. 2017, 126, 58-67.

(43) Grabs, I.-M.; Bradtmöller, C.; Menzel, D.; Garnweitner, G. Formation mechanisms of iron oxide nanoparticles in different nonaqueous media. Cryst. Growth Des. 2012, 12, 1469-1475.

(44) Stöber, W.; Fink, A.; Bohn, E. Controlled growth of monodisperse silica spheres in the micron size range. J. Colloid Interface Sci. 1968, 26, 62-69.

(45) Biedendieck, R.; Borgmeier, C.; Bunk, B.; Stammen, S.; Scherling, C.; Meinhardt, F.; Wittmann, C.; Jahn, D. Systems biology of recombinant protein production using Bacillus megaterium. Methods Enzymol. 2011, 500, 165-195. 